\author{
Michail Vikelis \\ Michail Xifaras \\ Georgios Magoufis \\ Georgios Gekas \\ Dimos Dimitrios Mitsikostas
}

\section{Headache attributed to unruptured saccular aneurysm, mimicking hemicrania continua}

Received: 14 January 2005

Accepted in revised form: 15 March 2005

Published online: 13 May 2005

M. Vikelis (西)

Androtsou 17,

Voula 16673, Greece

e-mail: m_vikelis@yahoo.co.uk

M. Vikelis • M. Xifaras • G. Gekas

Department of Neurology,

General Hospital of Nikea,

Nikea, Piraeus, Greece

M. Vikelis • D.D. Mitsikostas

Department of Neurology,

Athens Naval Hospital,

Athens, Greece

G. Magoufis

Department of Diagnostic and Interventional Neuroradiology,

Henry Dunant Hospital,

Athens, Greece

\begin{abstract}
Unruptured cerebral arterial aneurysms most often remain asymptomatic, but they may cause headache or other symptoms or signs. We describe herewith a case of headache attributed to an unruptured nternal carotid artery aneurysm, clearly mimicking the phenotype of hemicrania continua. Potential pathophysiological explanations and recommendations for recognition of similar cases are discussed.
\end{abstract}

Key words Hemicrania continua • Internal carotid artery aneurysm Unruptured aneurysm • Secondary headache

\section{Introduction}

Hemicrania continua ( $\mathrm{HC}$ ) is a rare primary headache disorder originally described by Sjaastad and Spierings in 1984 [1]. Clinical characteristics and diagnostic criteria include moderate, unilateral, continuous headache without side shift, exacerbations in the intensity of pain, autonomic features during pain exacerbations and absolute response to indomethacin [2]. Unruptured cerebral arterial aneurysms (CAAs) have a prevalence estimated at
$3.6 \%-6 \%$ of the general population [3]. They most often remain asymptomatic and represent an incidental finding at neuroimaging or autopsy, but they may present with headache, which is reported by up to one fifth of patients with unruptured CAAs [2]. In these cases headache usually has no specific features and may be accompanied by focal symptoms or signs [2-5].

We describe here a male patient with an unruptured internal carotid aneurysm that caused headache resembling HC. To the best of our knowledge this is the first report in the literature. 


\section{Case report}

A 35-year-old man presented with a 3-month history of continuous, mild, left-sided headache. He was a construction worker with free medical or family history and a heavy smoker (about 20 cigarettes per day, for the previous 15 years). Headache developed subacutely within a few days and was mild (2-5 on a $0-10$ visual scale) and continuous. Pain was maximal in the left orbital and temporal regions. There were exacerbations of severe pain (ranking 8-9/10), lasting a few hours and usually occurring early in the morning, once per day. During exacerbations autonomic features such as lacrimation, ptosis, miosis and nasal congestion followed by rhinorrhoea were present ipsilateral to the side of pain (Fig. 1). There was no headache aggravation or exacerbation triggering by usual physical activity such as climbing stairs, or nausea or photophobia/phonophobia at any time. Patient's clinical examination (both physical and neurological), routine blood tests and neuroimaging including brain computed tomography and magnetic resonance imaging (MRI) scan were all normal. Because the diagnosis of HC was likely, treatment was initiated with indomethacin $25 \mathrm{mg}$ three times daily and the dose was gradually increased up to 75

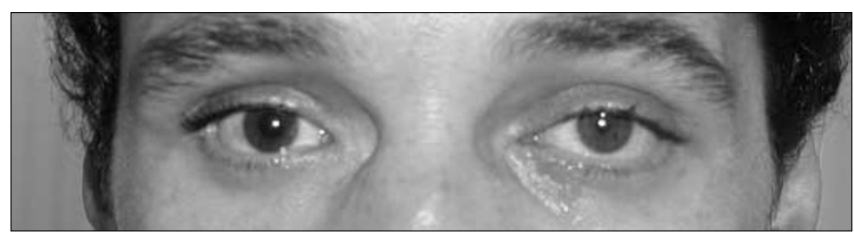

Fig. 1 Autonomic features during pain exacerbation. Miosis, eyelid ptosis and lacrimation can be observed in patient's left eye

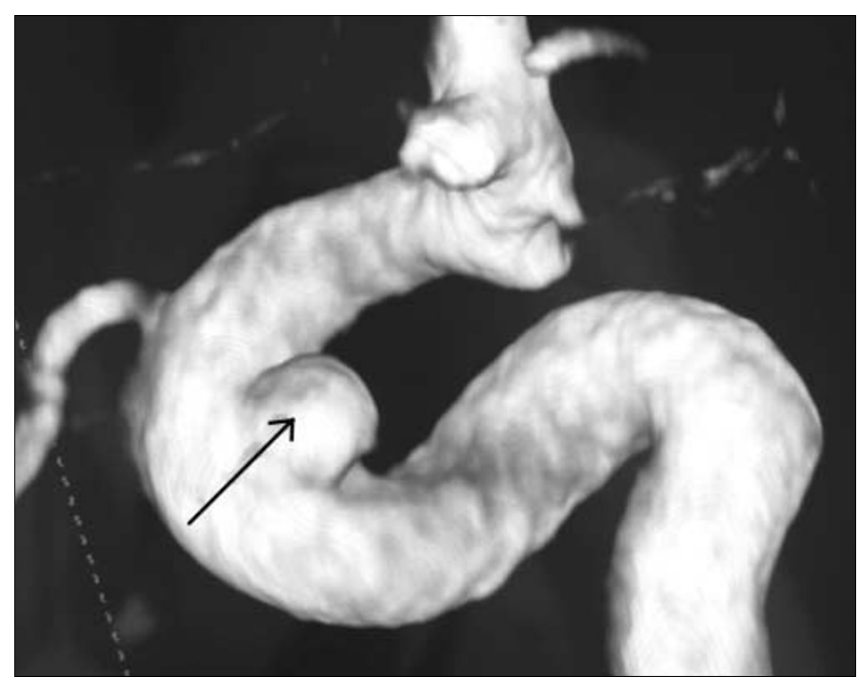

Fig. 2 Three-dimensional reconstruction of the digital subtraction angiography of the left internal carotid artery. The saccular aneurysm is indicated by an arrow mg twice daily as the response of baseline pain and of intensity and duration of exacerbations was good but not absolute (the baseline pain intensity under $150 \mathrm{mg}$ indomethacin daily was $1 / 10$ and the intensity of exacerbations 3-4/10). At this time a cerebral digital subtraction angiography was performed and revealed an aneurysm of the cavernous segment of the left internal carotid artery, measured 3x4 mm (Fig. 2). The patient was subsequently referred to an interventional neuroradiology centre, where the aneurysm was successfully treated by placement of a bare stent in front of the aneurysmal neck and occlusion of the aneurysmal sac with Guglielmi detachable coils. After treatment there was full headache remission and the patient remains headache free after 18 months.

\section{Discussion}

Unruptured CAAs most often remain asymptomatic, but they may manifest themselves through headache, which is reported by up to one fifth of patients with unruptured CAAs [2] and has no specific features in its onset, character or location [2-5]. In these cases focal symptoms, or signs, or seizures may accompany headache [5]. The pathophysiology of this headache remains elusive. Possible pathogenetic mechanisms include mass effect (caused by gradual or sudden growth of an aneurysm), intramural dissection within the aneurysmal wall and transmitted pulsations [6, 7]. To investigate the principal pathophysiological mechanism, Rodriguez-Catarino and colleagues retrospectively studied 10 cases of patients with unruptured symptomatic aneurysms treated by the endovascular route and found that other than the consistent increase in aneurysm volume after embolisation, no clinical deterioration was observed, thus concluding that mass effect may play a minor role in symptomatology of unruptured aneurysms and that transmitted pulsations may be more important [7].

In published cases of HC-like headache, infection and neoplasms, within or outside the cranium and head trauma in particular were described as associated pathologies [8-11]. In a recent report, a case of symptomatic HC associated with internal carotid artery dissection was described [12]. To distinguish the headache that is causally related with pathology from other headaches that are only coincidentally associated with it, Trucco and colleagues recently proposed specific criteria, including close temporal relationship between the associated disease and the onset of pain, side concordance, remission after surgical or aetiological therapy and prolonged post-treatment follow-up [13]. Our patient accomplishes the criteria of side concordance (both headache and the aneurysm 
were located within the left side), remission of headache after treatment of the primary pathology and prolonged post-treatment follow-up.

The consistent mild orbito-temporal pain with exacerbations accompanied with autonomic features in our patient was clearly mimicking the phenotype of HC [14]. Additionally, interictal neurological examination revealed no abnormal findings and a MRI brain scan result was normal as well. Our patient showed partial response to low doses of indomethacin. Although this is an 'ominous sign', raising suspicions for a possible organic aetiology [15], we consider the dose-dependent effect of indomethacin (by increasing the daily dose from 75 to 150 $\mathrm{mg}$ ) to be important. The "indotest" [16] was not applied because only per os formulations of indomethacin are available in Greece. The pattern of autonomic deficit that occurred during pain exacerbations in our patient resembled the deficit in patients with a postganglionic sympa- thetic lesion and is indicative of involvement of the cavernous carotid artery. The aneurysm could injure the traversing sympathetic fibres investing the arterial wall. It is difficult, however, to explain the temporal pattern of pain exacerbations and accompanying autonomic features.

In conclusion, this case demonstrates that a clinical presentation compatible with $\mathrm{HC}$ may be causatively related with intracranial pathology. This has been reported for other rare primary headache syndromes associated with autonomic features [13]. Thus, intensive paraclinical evaluation may be considered for any unusual onesided headache with recent onset and autonomic features, particularly when one or two of the diagnostic criteria for any primary headache syndrome are doubtful. A cerebral digital subtraction angiography or a magnetic resonance angiography should be included in the diagnostic workup of such patients, to rule out vascular underlying pathologies.

\section{References}

1. Sjaastad O, Spierings EHL (1984) 'Hemicrania continua'. Another headache absolutely responsive to indomethacin. Cephalalgia 4:65-70

2. Headache Classification Committee of the International Headache Society (2004) The International Classification of Headache Disorders, 2nd Edn. Cephalalgia 24[Suppl 1]:1-160

3. Wardlaw JM, White PM (2000) The detection and management of unruptured intracranial aneurysms. Brain 123:205-221

4. Raps EC, Rogers JD, Galetta SL, Solomon RA, Lennihan L, Klebanoff LM, Fink ME (1993) The clinical spectrum of unruptured intracranial aneurysms. Arch Neurol 50:265-268

5. Weir B (1994) Headaches from aneurysms. Cephalalgia 14:79-87

6. Linn FH, Rinkel GJE, Algra A, van Gijn J (2000) The notion of "warning leaks" in subarachnoid haemorrhage: are such patients in fact admitted with a rebleed? J Neurol Neurosurg Psychiatry 68:332-336
7. Rodriguez-Catarino M, Frisen L, Wikholm G, Elfverson J, Quiding L, Svendsen P (2003) Internal carotid artery aneurysms, cranial nerve dysfunction and headache: the role of deformation and pulsation.

Neuroradiology 45:236-240

8. Meckling SK, Becker WJ (1997) Sphenoid sinusitis presenting as indomethacin-responsive 'hemicrania continua': a case report. Cephalalgia 17:303

9. Antonaci F, Sjaastad O (1992) Hemicrania continua: a possible symptomatic case, due to a mesenchymal tumor. Funct Neurol 7:471-474

10. Erros EJ, Swanson JW, Dodick DW (2002) Hemicrania continua: an indomethacin responsive case with an underlying malignant etiology. Headache 42:527-529

11. Lay CL, Newman LC (1999) Posttraumatic hemicrania continua. Headache 39:275-279
12. Rogalewski A, Evers S (2005) Symptomatic hemicrania continua after internal carotid artery dissection. Headache 45:167-169

13. Trucco M, Mainardi F, Maggioni F, Badino R, Zanchin G (2004) Chronic paroxysmal hemicrania, hemicrania continua and SUNCT syndrome in association with other pathologies: a review. Cephalalgia 24:173-184

14. Rapoport AM, Bigal ME (2003) Hemicrania continua: clinical and nosographic update. Neurol Sci 24[Suppl 2]:118-121

15. Sjaastad O, Stovner LJ, Stolt-Nielsen A, Antonaci F, Fredriksen TA (1995) $\mathrm{CPH}$ and hemicrania continua: requirements of high indomethacin dosages an ominous sign? Headache 35:363-367

16. Antonaci F, Pareja JA, Caminero AB, Sjaastad O (1998) Chronic paroxysmal hemicrania and hemicrania continua. Parenteral indomethacin: the 'indotest'. Headache 38:122-128 